\title{
基于硝基还原机理的一氧化碳荧光探针的开发及细胞成像研究
}

\author{
陈恩庆 ${ }^{a}$ 唐永和 ${ }^{a}$ 王 蕾 ${ }^{b}$ 任江波 ${ }^{a}$ 林伟英 $*, a$ \\ $\left({ }^{a}\right.$ 广西电化学能源材料重点实验室 广西大学光功能材料与化学生物学研究院 \\ 广西大学化学化工学院 南宁 530004) \\ ( $b$ 南宁市食品药品检验所 南宁 530007)
}

\begin{abstract}
摘要 一氧化碳 $(\mathrm{CO})$ 作为一种重要的细胞气体信号传递分子, 参与生物体内重要的生理和病理功能. 选用经典的苂光 平台一一菜酰亚胺荧光平台，以硝基基团作为识别位点，通过严谨的设计和两步合成的方法，开发了一种新型用来检 测一氧化碳的苂光增强型荧光探针 NaLy-CO. 光谱测试数据表明, 该苂光探针对一氧化碳具有较好的选择性和灵敏 度. 利用苂光共聚焦成像技术，成功地对细胞外源性和内源性一氧化碳进行了检测. 此外，共定位成像实验表明，该荧 光探针可以用于检测细胞溶酶体中的一氧化碳.
\end{abstract}

关键词 一氧化碳; 苂光探针; 生物成像; 硝基; 萗酰亚胺

\section{Development of a New Carbon Monoxide Fluorescent Probe Based on Nitro Reduction and Its Bioimaging Research in Living Cells}

\author{
Chen, Enqing $^{a} \quad$ Tang, Yonghe $^{a} \quad$ Wang, Lei $^{b} \quad$ Ren, Jiangbo ${ }^{a} \quad$ Lin, Weiying*,a \\ $\left({ }^{a}\right.$ Guangxi Key Laboratory of Electrochemical Energy Materials, Institute of Optical Materials and Chemical Biology, \\ School of Chemistry and Chemical Engineering, Guangxi University, Nanning 530004) \\ ( ${ }^{b}$ Nanning Institute for Food and Drug Control, Nanning 530007)
}

\begin{abstract}
Carbon monoxide (CO), as an important cell signaling molecule, is involved in important physiological and pathological functions in organism. A new fluorescence enhanced CO fluorescent probe NaLy-CO was developed by using naphthalimide dye as fluorescence platform and nitro group as the recognition site. The results of spectrum test in solution showed that the fluorescent probe NaLy-CO had good selectivity and sensitivity to CO. The fluorescent probe NaLy-CO was successfully used to detect exogenous and endogenous $\mathrm{CO}$ in living HeLa cells by using confocal fluorescence imaging technology. In addition, the colocation imaging experiments showed that the fluorescent probe NaLy-CO could detect CO in the lysosome of living cells.

Keywords carbon monoxide; fluorescent probe; bioimaging; nitro; naphthalimide
\end{abstract}

众所周知, 一氧化碳 $(\mathrm{CO})$ 是一种无色无味的气体. 一氧化碳进入生物体, 会与血红蛋白中心的铁离子进行 配位形成碳氧血红蛋白, 进而阻碍血红蛋白与氧分子结 合, 造成氧气的正常运输受阻, 从而导致生物组织缺氧 室息 ${ }^{[1]}$, 所以很长一段时间内, $\mathrm{CO}$ 都被认为是一种有毒 气体. 随着生物医学技术的发展, 研究人员发现 $\mathrm{CO}$ 能 够在体内自发产生, 例如血红素在血红素氧合酶( $\mathrm{HO})$
的催化下会产生内源性 $\mathrm{CO}^{[2]}$. 此外, $\mathrm{CO}$ 还承担着生物 体内包括抑制血小板聚集 ${ }^{[3]}$ 、呼吸调节 ${ }^{[4]}$ 以及体温调节 ${ }^{[5]}$ 等在内的重要的生理病理过程, 同时也参与一些疾病的 治疗过程 ${ }^{[6-7]}$. 因此, $\mathrm{CO}$ 被认为是一种既有毒性作用, 又有生物活性的气体, 是生命过程中重要的气体信号分 子之一. 然而内源性的 $\mathrm{CO}$ 浓度异常与心血管疾病、高 血压、炎症、肥胖、癌症等多种疾病的发生和恶化密切

* Corresponding author. E-mail: weiyinglin2013@163.com

Received September 7, 2020; revised October 6, 2020; published online October 27, 2020.

Project supported by the National Natural Science Foundation of China (Nos. 21672083, 21877048, 22077048), the Startup Fund of Guangxi University (No. A3040051003) and the Natural Science Foundation of Guangxi Province (No. 2019GXNSFBA245068).

国家自然科学基金(Nos. 21672083, 21877048, 22077048)、广西大学启动基金(No. A3040051003)和广西省自然科学基金(Nos. 2019GXNSFBA245068) 资助项目. 
相关 ${ }^{[8-12]}$. 因此, 设计新的方法来选择性地、可视化地研 究一氧化碳在活体系统中的生理、病理功能是很重要的.

在真核细胞中, 溶酶体是一种普遍存在的细胞器, 它内含数十种生物酶(例如水解酶、硫酸酯酶和蛋白酶 等), 参与细胞内的消化代谢、免疫应激及酶加工等重要 的生理过程 ${ }^{[13-14]}$. 许多生物活性分子都存在于溶酶体 中, 如活性氧和活性氮等, 对溶酶体内的活性小分子进 行实时监测, 将为研究与溶酶体相关的疾病的发生、发 展以及治疗等提供不可忽视的理论支撑. 溶酶体被认作 细胞器中的 “清道夫”, 过多的 CO 进入细胞后被传递 至溶酶体中 ${ }^{[15-16]}$. 但是, 目前为止, $\mathrm{CO}$ 在溶酶体内所扮 演的这种 “毒性/信号” 的双重角色的界限仍然模糊不清, 因此检测溶酶体中内源性 $\mathrm{CO}$ 仍具有很大的挑战性.

传统的检测 $\mathrm{CO}$ 的方法, 例如显色法、吸收光谱法、 色谱法和电化学分析等 ${ }^{[17-19]}$, 需要破坏被测样品, 无法 实现 $\mathrm{CO}$ 的原位检测. 相比之下, 荧光显微成像技术因 其能够实现生物活性分子的原位实时检测而备受关

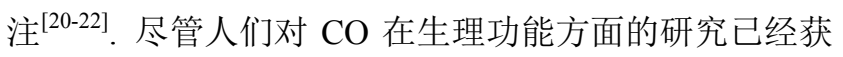
得了一些成果 ${ }^{[23]}$, 但目前还没有能够检测溶酶体内源 性 CO 的苂光探针, 对溶酶体内源性 CO 所发挥作用的 研究仍是目前的一大难题.

因此, 开发一种可以用来检测细胞溶酶体内源性 $\mathrm{CO}$ 的荧光探针极具研究价值. 本工作设计并合成了新 型 CO 苂光探针 NaLy-CO. 研究表明探针 NaLy-CO 对 $\mathrm{CO}$ 具有较好的选择性和灵敏度. 除此之外, 利用荧光 共聚焦成像技术, 研究了探针 NaLy-CO 对细胞的内源 性和外源性 CO 的成像. 此外, 共定位成像实验表明, 探针 NaLy-CO 能够检测细胞溶酶体内的 CO.

\section{1 结果与讨论}

\section{1 荧光探针 NaLy-CO 的设计策略及合成过程}

硝基由于拥有极强的吸电子能力, 可以对探针的电 子云进行调控. 此外, $\mathrm{CO}$ 作为易失去电子的还原剂, 可 以将硝基基团还原成氨基基团 ${ }^{[24]}$. 基于上述设计策略, 通过在萗酰亚胺苂光平台上引入 $\mathrm{CO}$ 识别位点——硝基 基团, 以及溶酶体靶向基团一一吡啶基团, 构建出了新 型 CO 苂光探针 NaLy-CO. 硝基作为吸电子基团, 阻碍 了分子内电荷转移(ICT)效应, 因此探针 NaLy-CO 几乎 没有荧光信号产生. 当探针 NaLy-CO 与 CO 相遇发生 反应后, 硝基被还原为氨基, 此时分子的电子结构表现 为 “推-拉” 电子体系, 恢复 ICT 效应, 从而产生强烈的 荧光信号(Scheme 1).

探针是经过两步反应合成得到的(Scheme 2). 以 1,8 -荎二酸䣶为原料, 通过硝化反应得到中间体 $\mathbf{1}^{[25]}$. 然 后中间体 1 在乙醇中与(2-氨基乙基)吡啶加热回流得到

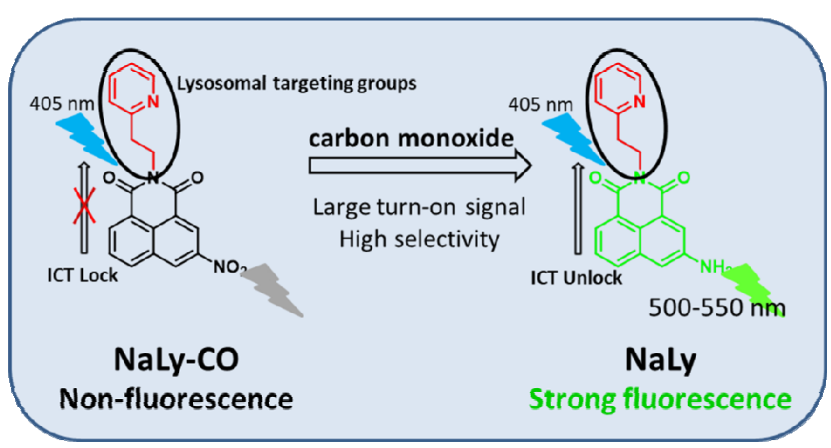

图式 1 探针 NaLy-CO 对 CO 的识别机制

Scheme 1 Sensing mechanism of probe NaLy-CO to CO

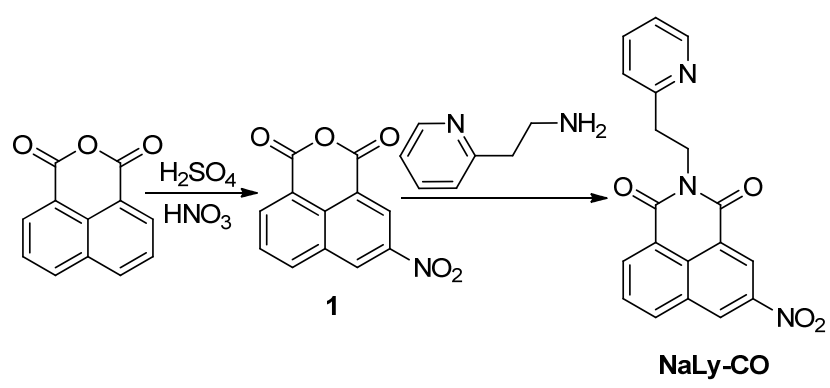

图式 2 探针 NaLy-CO 的合成路线

Scheme 2 Synthetic route of probe NaLy-CO

探针 NaLy-CO. 利用核磁(氢谱、碳谱)和高分辨质谱对 所合成的化合物进行了结构表征.

\section{2 探针 NaLy-CO 对 CO 的苂光滴定光谱研究}

合成苂光探针 NaLy-CO 并确定其结构之后, 进行 了探针和 $\mathrm{CO}$ 相互作用的荧光光谱测试. $37{ }^{\circ} \mathrm{C}$ 条件下, $1 \times 10^{-5} \mathrm{~mol} / \mathrm{L}$ 探针和不同浓度的 CORM-2 (三羰基二氯 代钓二聚物, $\mathrm{CO}$ 释放试剂, $0 \sim 5 \times 10^{-4} \mathrm{~mol} / \mathrm{L}$ ) 在磷酸缓 冲溶液(PBS, pH 7.4, $1 \times 10^{-2} \mathrm{~mol} / \mathrm{L}$ ) 作用 $40 \mathrm{~min}$ 后进行 荧光光谱测试, 结果如图 1 所示. 和我们预期的一样, 单独探针的苂光微乎其微. 当添加 $\mathrm{CO}$ 后, 测试体系中 的苂光强度将随 $\mathrm{CO}$ 的浓度的增加逐渐增强. 通过数据 整合, 选取 $523 \mathrm{~nm}$ 处的苂光强度作图(强度随 $\mathrm{CO}$ 浓度 的变化曲线), 可以看出, $\mathrm{CO}$ 浓度在 $0 \sim 1 \times 10^{-5} \mathrm{~mol} / \mathrm{L}$ 范围内, 测试体系的苂光强度具有较好的线性关系(线 性相关系数 $R^{2}=0.9966$ )(图 2). 对探针的检测限进行计 算 $\left(3 \sigma / \kappa^{\text {法 }}{ }^{[26]}\right)$, 探针对 $\mathrm{CO}$ 的检出限为 $7.90 \times 10^{-8} \mathrm{~mol} / \mathrm{L}$. 这些数据表明, 该荧光探针 NaLy-CO 对 CO 具有较高 的信噪比和灵敏度, 适用于检测样品中微量的 CO.

\section{3 探针 NaLy-CO 对 CO 的荧光动力学及探针光稳定 性能研究}

随后，进行了探针 NaLy-CO 与 CO 相互作用的时间 依赖性研究. 探针 $\left(1 \times 10^{-5} \mathrm{~mol} / \mathrm{L}\right)$ 和不同浓度的 CORM$2\left(2.5 \times 10^{-4} 、 2 \times 10^{-4} 、 1 \times 10^{-4} 、 5 \times 10^{-5} \mathrm{~mol} / \mathrm{L}\right)$ 在 PBS 缓冲溶液里相互作用. 如图 3 所示, 随着探针与 $\mathrm{CO}$ 作 


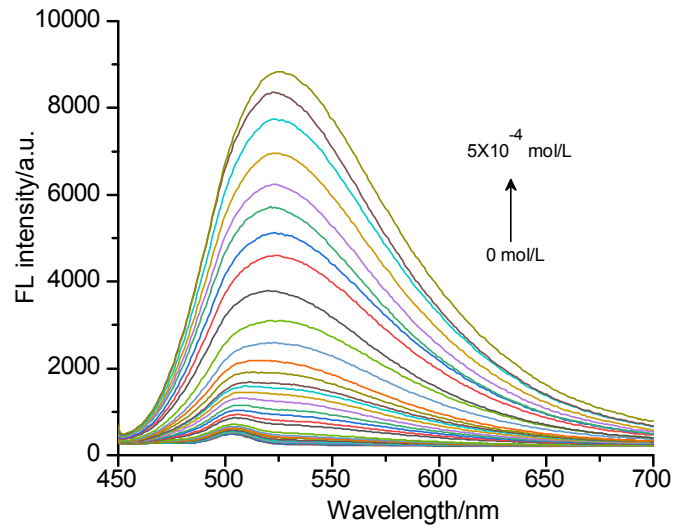

图 1 探针 NaLy-CO $\left(1 \times 10^{-5} \mathrm{~mol} / \mathrm{L}\right)$ 与 CORM-2 $\left(0 \sim 5 \times 10^{-4}\right.$ $\mathrm{mol} / \mathrm{L})$ 相互作用的苂光曲线

Figure 1 Fluorescent curves of probe NaLy-CO $\left(1 \times 10^{-5}\right.$ $\mathrm{mol} / \mathrm{L})$ encountering with CORM-2 $\left(0 \sim 5 \times 10^{-4} \mathrm{~mol} / \mathrm{L}\right)$

In $1 \times 10^{-2} \mathrm{~mol} / \mathrm{L}$ PBS buffer (pH 7.4, $10 \mathrm{vol} \%$ DMSO) for $40 \mathrm{~min} . \lambda_{\mathrm{ex}}=$ $430 \mathrm{~nm}$

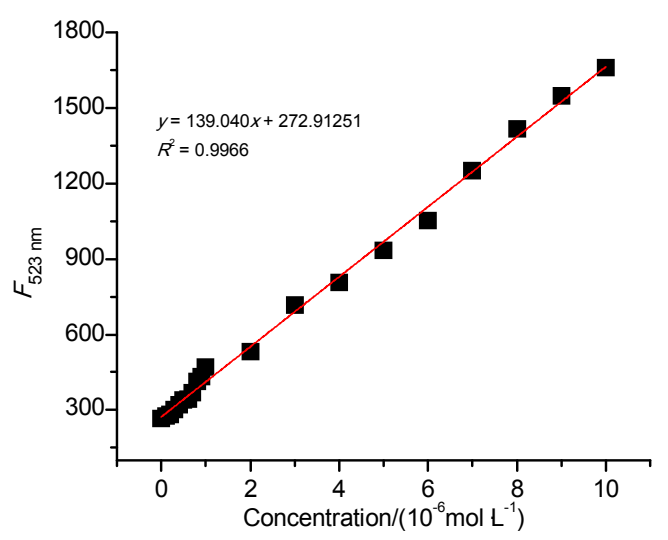

图 2 探针 NaLy-CO 在 $523 \mathrm{~nm}$ 处苂光强度与不同浓度的 CORM-2 $\left(0 \sim 1 \times 10^{-5} \mathrm{~mol} / \mathrm{L}\right)$ 的线性关系

Figure 2 Linear relationship between the fluorescence intensity of the probe NaLy-CO and the concentration of CORM-2 ( $0 \sim$ $1 \times 10^{-5} \mathrm{~mol} / \mathrm{L}$ ) at $523 \mathrm{~nm}$

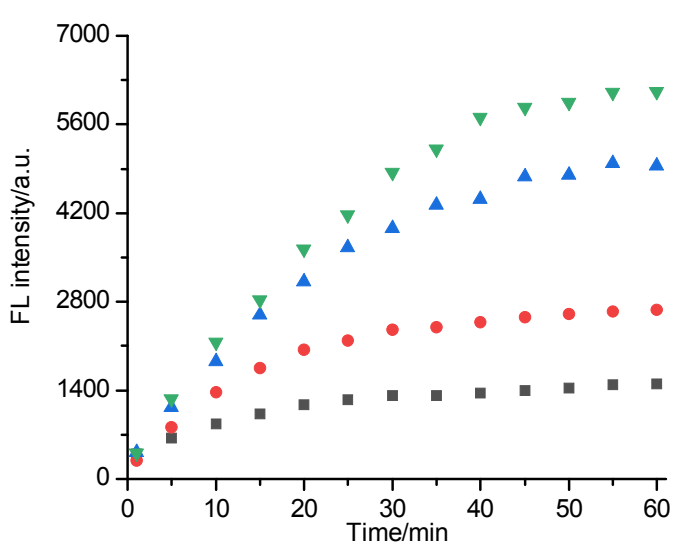

图 3 探针 NaLy-CO 与 CORM-2 相互作用的荧光强度随时间 变化曲线

Figure 3 Time-dependent fluorescent of probe NaLy-CO intensities at $F_{523 \mathrm{~nm}}$ treated with CORM-2

$\nabla 2.50 \times 10^{-4} \mathrm{~mol} / \mathrm{L}, \Delta 2 \times 10^{-4} \mathrm{~mol} / \mathrm{L}, \bullet 1 \times 10^{-4} \mathrm{~mol} / \mathrm{L}, \square 5 \times 10^{-5}$ $\mathrm{mol} / \mathrm{L} \cdot \lambda_{\mathrm{ex}}=430 \mathrm{~nm}$
用时间的增加，在 $523 \mathrm{~nm}$ 处的苂光强度有着明显的增 强，大约 $40 \mathrm{~min}$ 后达到平衡状态。在测试探针 NaLy-CO 的光稳定性过程中, 选择 PBS 作为缓冲液. 如图 4 所示, 对探针 NaLy-CO 持续使用 $365 \mathrm{~nm}$ 的紫外 线进行照射，每隔一段时间监测 $523 \mathrm{~nm}$ 处的荧光强度， 可以发现其苂光强度几乎没有变化. 这些数据表明, 作 为一种苂光检测试剂, 探针 NaLy-CO 对 CO 具有良好的 识别速度和良好的光稳定性.

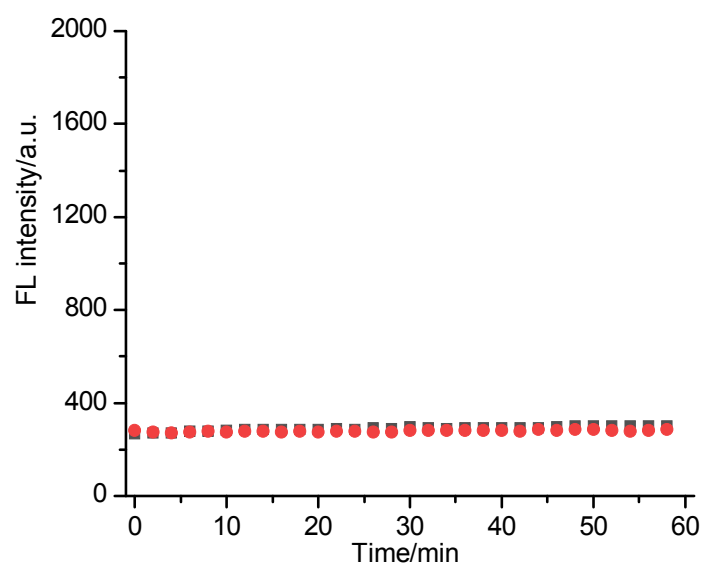

图 4 探针 NaLy-CO $\left(1 \times 10^{-5} \mathrm{~mol} / \mathrm{L}\right)$ 的光稳定性能 Figure 4 Photo-stability profiles of probe NaLy-CO $\left(1 \times 10^{-5}\right.$ $\mathrm{mol} / \mathrm{L}$ )

Photo-stability profiles of the probe NaLy-CO $\left(1 \times 10^{-5} \mathrm{~mol} / \mathrm{L}\right)$ in the absence $(\boldsymbol{\square})$ or presence of UV-irradiated $(-)(365 \mathrm{~nm})$

\section{4 探针 NaLy-CO 对不同分析物的选择性研究}

探针优异的选择性，是进行有效检测目标分子的必 要因素之一. 因此又进行了探针 NaLy-CO 的选择性识 别测试. 选用 $1 \times 10^{-5} \mathrm{~mol} / \mathrm{L}$ 探针和不同的生物相关分 析物在 PBS 缓冲溶液相互作用 $30 \mathrm{~min}$ 后进行苂光发射 光谱测试, 并记录在 $523 \mathrm{~nm}$ 处的苂光强度变化. 这些分 析物分别是谷胱甘肽 $\left(\mathrm{GSH}, 5 \times 10^{-3} \mathrm{~mol} / \mathrm{L}\right) 、 L$-半胱氨酸 $\left(L\right.$-Cys, $\left.1 \times 10^{-3} \mathrm{~mol} / \mathrm{L}\right)$ 、甘氨酸 $\left(\mathrm{Gly}, 5 \times 10^{-4} \mathrm{~mol} / \mathrm{L}\right)$ 、 $D L$ - 同型半胱氨酸 $\left(D L-\mathrm{Hcy}, 5 \times 10^{-4} \mathrm{~mol} / \mathrm{L}\right)$ 、葡萄糖 (glucose, $5 \times 10^{-4} \mathrm{~mol} / \mathrm{L}$ )、 $L$-抗坏血酸(Ascorbic Acid, $\left.5 \times 10^{-4} \mathrm{~mol} / \mathrm{L}\right)$ 、丙酮(acetone, $\left.5 \times 10^{-4} \mathrm{~mol} / \mathrm{L}\right)$ 、丙酮酸 (pyruvic acid, $5 \times 10^{-4} \mathrm{~mol} / \mathrm{L}$ )、丙酮酸钠(pyruvic acid sodium, $\left.5 \times 10^{-4} \mathrm{~mol} / \mathrm{L}\right)$ 、次氯酸钠 $\left(\mathrm{NaClO}, 5 \times 10^{-4}\right.$ $\mathrm{mol} / \mathrm{L})$ 、过氧化氢 $\left(\mathrm{H}_{2} \mathrm{O}_{2}, 5 \times 10^{-4} \mathrm{~mol} / \mathrm{L}\right)$ 、甲醛 $(\mathrm{FA}, 5 \times$ $\left.10^{-4} \mathrm{~mol} / \mathrm{L}\right)$ 、硫化钠 $\left(\mathrm{Na}_{2} \mathrm{~S}, 5 \times 10^{-4} \mathrm{~mol} / \mathrm{L}\right)$ 、硫氢化钠 $\left(\mathrm{NaHS}, 5 \times 10^{-4} \mathrm{~mol} / \mathrm{L}\right) 、$ 硫氧化钠 $\left(\mathrm{NaSCN}, 5 \times 10^{-4}\right.$ $\mathrm{mol} / \mathrm{L})$ 、叔丁基过氧化氢 $\left(\mathrm{TBHP}, 5 \times 10^{-4} \mathrm{~mol} / \mathrm{L}\right)$ 、硝普 酸钠 $\left(\mathrm{SNP}, 5 \times 10^{-4} \mathrm{~mol} / \mathrm{L}\right)$ 、亚硫酸钠 $\left(\mathrm{Na}_{2} \mathrm{SO}_{3}, 5 \times 10^{-4}\right.$ $\mathrm{mol} / \mathrm{L})$ 、亚硫酸氢钠 $\left(\mathrm{NaHSO}_{3}, 5 \times 10^{-4} \mathrm{~mol} / \mathrm{L}\right)$ 、亚硝酸 钠 $\left(\mathrm{NaNO}_{2}, 5 \times 10^{-4} \mathrm{~mol} / \mathrm{L}\right) 、 乙 二$ 醛 $\left(\mathrm{GO}, 5 \times 10^{-4} \mathrm{~mol} / \mathrm{L}\right)$ 和 CORM-2 (CO, $\left.1 \times 10^{-4} \mathrm{~mol} / \mathrm{L}\right)$. 如图 5 所示, 除了加 入 CO 外, 其它分析物对探针 NaLy-CO 的苂光强度几乎 
没有变化. 对比其他的分析物, $\mathrm{CO}$ 的引入引起探针荧光 强度明显的变化. 该实验说明该苂光探针 NaLy-CO 对 $\mathrm{CO}$ 具有很强的选择性, 有望在复杂的生物环境中检测 $\mathrm{CO}$.

\section{5 探针 NaLy-CO 对 CO 的识别机理研究}

拥有极强的吸电子能力的硝基可以调控荧光探针 中电子云的分布. 此外, 当 $\mathrm{CO}$ 等还原物存在的情况下, 硝基将会被还原成氨基. 我们利用高分辨质谱来验证探 针 NaLy-CO 对 CO 的识别机理. $1 \times 10^{-5} \mathrm{~mol} / \mathrm{L}$ 探针 NaLy-CO 和 $1 \times 10^{-4} \mathrm{~mol} / \mathrm{L}$ CORM-2 在二甲基亚砜和水 $(V: V=8: 2)$ 混合溶剂里孵育 $30 \mathrm{~min}$ 后进行高分辨质 谱测试. 测试结果显示出现了数值为 318.1237 的分子离 子峰, 该数值与探针 NaLy-CO 的还原产物的理论分子 离子峰 $\left([\mathrm{M}+\mathrm{H}]^{+} 318.1237\right)$ 相吻合. 高分辨质谱测试结 果数据表明探针 NaLy-CO 的硝基基团可以被 $\mathrm{CO}$ 还原. 1.6 探针 NaLy-CO 在不同 $\mathrm{pH}$ 溶液中对 $\mathrm{CO}$ 的识别研 究

探针 NaLy-CO 作为一种生物活性分子示踪剂, 在 生理 $\mathrm{pH}$ 值下应对目标活性小分子有显著的识别能力. 为了进一步说明探针 NaLy-CO 在生理 $\mathrm{pH}$ 环境下对 CO 的检测能力, 在不同 $\mathrm{pH}$ 值 $(4.5 \sim 10.0)$ 的 PBS 缓冲溶液 中, 分别对探针、探针和 $\mathrm{CO}$ 作用后的荧光强度进行检 测. 如图 6 所示, 单纯探针 NaLy-CO $\left(1 \times 10^{-5} \mathrm{~mol} / \mathrm{L}\right)$ 在 不同 $\mathrm{pH}$ 值的 PBS 缓冲溶液中的荧光发射强度变化不大, 说明探针 NaLy-CO 的稳定性不受酸度和碱度的影响. 探针 NaLy-CO $\left(1 \times 10^{-5} \mathrm{~mol} / \mathrm{L}\right)$ 与 CO $\left(1 \times 10^{-4} \mathrm{~mol} / \mathrm{L}\right)$ 相互作用后, 其荧光强度在不同 $\mathrm{pH}$ 条件下有显著变化,
这表明探针 NaLy-CO 可以在不同的酸碱环境中对 CO 进行检测, 说明探针有着较为广泛的使用范围.

荧光光谱测试表明，该探针 NaLy-CO 具有较高的 灵敏度和选择性，优异的光稳定性，能在不同 $\mathrm{pH}$ 值溶 液中稳定存在和识别 CO. 探针 NaLy-CO 的上述优良光 学行为将促进进一步的生物成像研究的开展.

\section{7 探针 NaLy-CO 的生物成像研究}

荧光探针是一种可以定位追踪的生物活性分子跟 踪剂, 拥有低毒性和优异的生物相容性是一个合格探针 的必要条件. 噻唑蓝(MTT) 法检测数据显示, 探针 NaLy-CO 在不同浓度 $\left(0 \sim 5 \times 10^{-5} \mathrm{~mol} / \mathrm{L}\right)$ 下对 HeLa 细 胞无明显毒性, 说明探针 NaLy-CO 可以用于生物样品 中 $\mathrm{CO}$ 的检测.

在确定探针 NaLy-CO 没有明显的细胞毒性后, 开 始用探针 NaLy-CO 进行细胞内 CO 的成像研究. 首先, 为了验证探针 NaLy-CO 可以在复杂的生理环境中对 $\mathrm{CO}$ 进行检测识别, 通过外添加 $\mathrm{CO}$ 的方法进行细胞的 荧光成像. 如图 7 所示, 用 $1 \times 10^{-5} \mathrm{~mol} / \mathrm{L}$ 探针 $\mathbf{N a L y}-$ CO (图 7B) 或 $1 \times 10^{-4} \mathrm{~mol} / \mathrm{L}$ CORM-2 (图 7E) 捊育细胞 $30 \mathrm{~min}$ 后进行荧光成像, 并没有荧光信号产生. 与两组 实验现象形成鲜明对比的是, HeLa 细胞与 $1 \times 10^{-4}$ $\mathrm{mol} / \mathrm{L} \mathrm{CORM}-2$ 捊育 $30 \mathrm{~min}$ 后继续用 $1 \times 10^{-5} \mathrm{~mol} / \mathrm{L}$ 探 针 NaLy-CO 孵育 $30 \mathrm{~min}$ 后进行荧光成像, 此时检测到 了强烈的绿色荧光信号(图 7H). 外加的 $\mathrm{CO}$ 成像实验结 果与光谱实验结果一致, 说明该苂光探针可以稳定地存 在于复杂的细胞环境中, 并且该探针 NaLy-CO 具有跟 踪细胞外源性 CO 的能力.

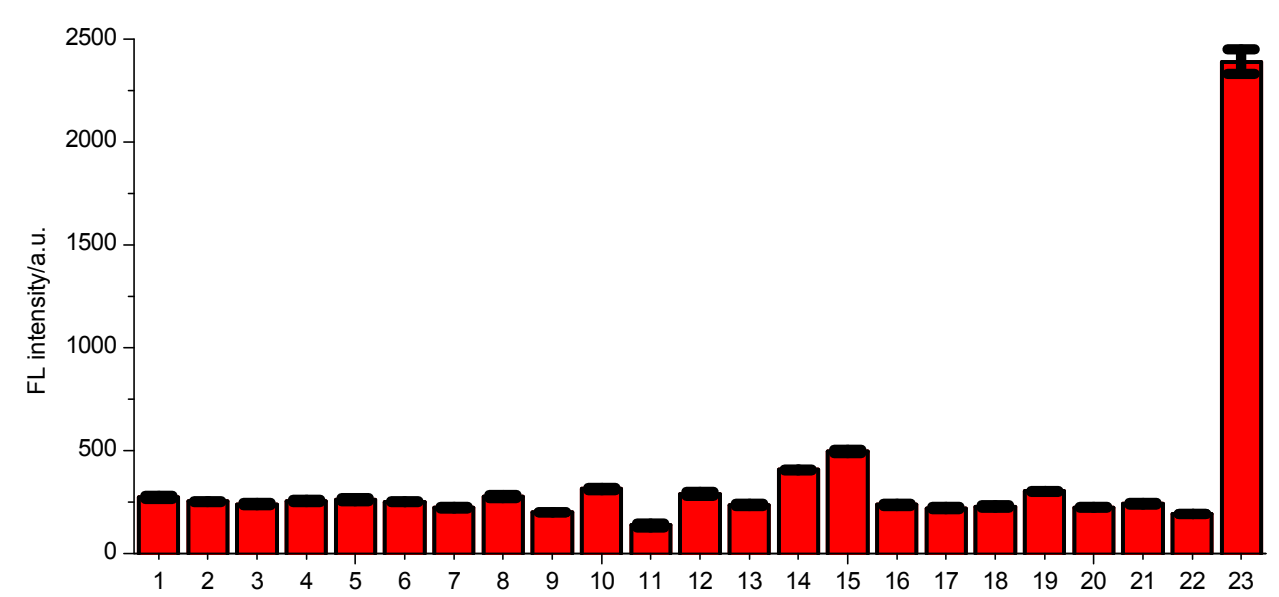

图 5 探针 NaLy-CO 与各种分析物相互作用后在 $523 \mathrm{~nm}$ 处的荧光强度变化

Figure 5 Fluorescent intensity changes of the probe NaLy-CO interacts with various analytes at $523 \mathrm{~nm}$ The numbers represent: $(1)$ probe $\left(1 \times 10^{-5} \mathrm{~mol} / \mathrm{L}\right),(2)$ glucose $\left(5 \times 10^{-4} \mathrm{~mol} / \mathrm{L}\right),(3) \mathrm{L}$-Cys $\left(1 \times 10^{-3} \mathrm{~mol} / \mathrm{L}\right),(4)$ ascorbic acid $\left(5 \times 10^{-4} \mathrm{~mol} / \mathrm{L}\right),(5)$ acetone $\left(5 \times 10^{-4} \mathrm{~mol} / \mathrm{L}\right),(6)$ pyruvic acid $\left(5 \times 10^{-4} \mathrm{~mol} / \mathrm{L}\right)$, (7) pyruvic acid sodium $\left(5 \times 10^{-4} \mathrm{~mol} / \mathrm{L}\right),(8) \mathrm{NaClO}\left(5 \times 10^{-4} \mathrm{~mol} / \mathrm{L}\right),(9) \mathrm{Gly}\left(5 \times 10^{-4} \mathrm{~mol} / \mathrm{L}\right)$, (10) $D L$-Hcy $\left(5 \times 10^{-4} \mathrm{~mol} / \mathrm{L}\right),(11) \mathrm{GSH}\left(5 \times 10^{-3} \mathrm{~mol} / \mathrm{L}\right),(12) \mathrm{H}_{2} \mathrm{O}_{2}\left(5 \times 10^{-4} \mathrm{~mol} / \mathrm{L}\right),(13) \mathrm{FA}\left(5 \times 10^{-4} \mathrm{~mol} / \mathrm{L}\right),(14) \mathrm{Na}_{2} \mathrm{~S}\left(5 \times 10^{-4} \mathrm{~mol} / \mathrm{L}\right),(15) \mathrm{NaHS}$ $\left(5 \times 10^{-4} \mathrm{~mol} / \mathrm{L}\right),(16) \mathrm{NaSCN}\left(5 \times 10^{-4} \mathrm{~mol} / \mathrm{L}\right),(17) \mathrm{TBHP}\left(5 \times 10^{-4} \mathrm{~mol} / \mathrm{L}\right),(18) \mathrm{SNP}\left(5 \times 10^{-4} \mathrm{~mol} / \mathrm{L}\right),(19) \mathrm{Na}_{2} \mathrm{SO}_{3}\left(5 \times 10^{-4} \mathrm{~mol} / \mathrm{L}\right),(20) \mathrm{NaHSO}$ $\left(5 \times 10^{-4} \mathrm{~mol} / \mathrm{L}\right),(21) \mathrm{NaNO}_{2}\left(5 \times 10^{-4} \mathrm{~mol} / \mathrm{L}\right),(22) \mathrm{GO}\left(5 \times 10^{-4} \mathrm{~mol} / \mathrm{L}\right),(23) \mathrm{CORM}-2\left(1 \times 10^{-4} \mathrm{~mol} / \mathrm{L}\right) . \lambda_{\mathrm{ex}}=430 \mathrm{~nm}$ 


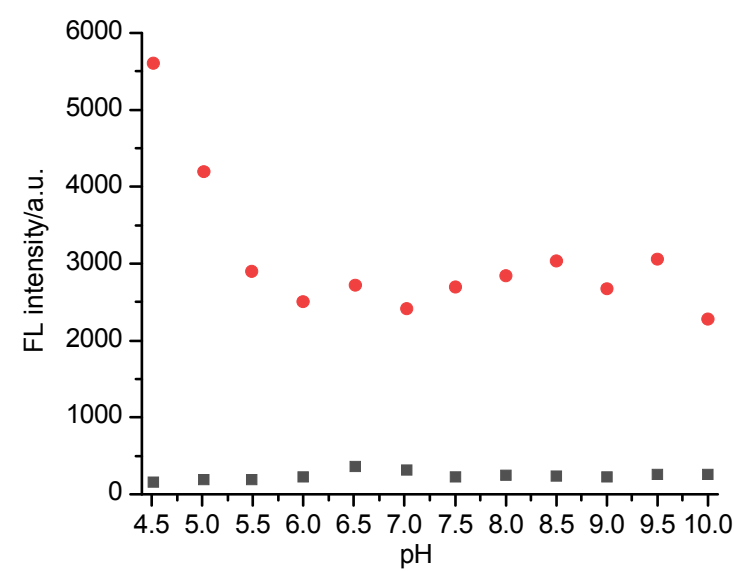

图 6 探针 NaLy-CO 和 CORM-2 在不同 $\mathrm{pH}$ 溶液里发生反应 后在 $523 \mathrm{~nm}$ 的苂光强度

Figure 6 Fluorescence intensity of probe NaLy-CO and CORM-2 in different $\mathrm{pH}$ solutions at $523 \mathrm{~nm}$

Fluorescence intensity changes of the probe NaLy-CO $\left(1 \times 10^{-5} \mathrm{~mol} / \mathrm{L}\right)$ at different $\mathrm{pH}$ values in the absence $(\Theta)$ or presence $(\square)$ of $\mathrm{CO}(1 \times$ $10^{-4} \mathrm{~mol} / \mathrm{L}$ ) for $40 \mathrm{~min}$
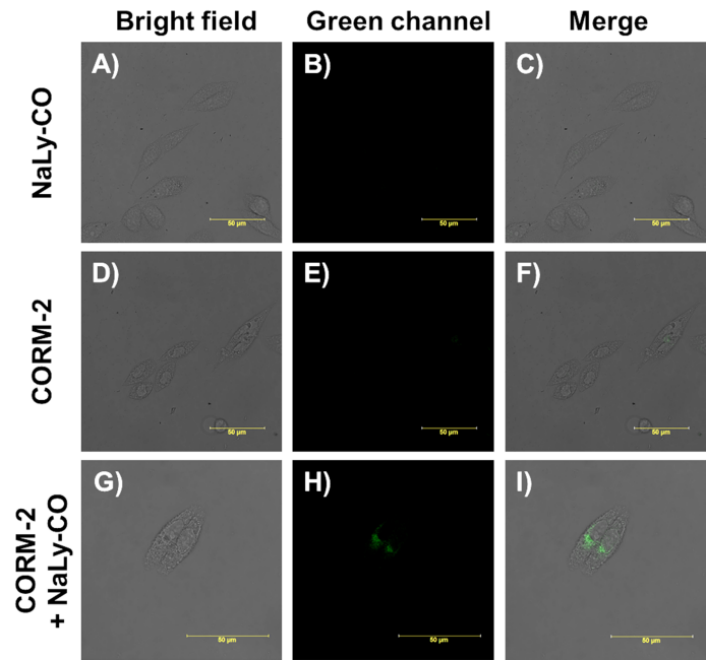

图 7 探针 NaLy-CO 在 HeLa 细胞中检测外源性 CO 的荧光

图像

Figure 7 Fluorescent images of probe NaLy-CO in HeLa cells for detecting exogenous $\mathrm{CO}$

$\mathrm{A} \sim \mathrm{C}$ : the $\mathrm{HeLa}$ cells were incubated with $1 \times 10^{-5} \mathrm{~mol} / \mathrm{L}$ probe NaLy-CO for $30 \mathrm{~min}$; D F : the HeLa cells were incubated with $1 \times 10^{-}$ ${ }^{4} \mathrm{~mol} / \mathrm{L}$ CORM-2 for $30 \mathrm{~min}$; $\mathrm{G} \sim \mathrm{I}$ : the HeLa cells were pre-cultured with $1 \times 10^{-4} \mathrm{~mol} / \mathrm{L}$ CORM- 2 for $30 \mathrm{~min}$, then cultured with $1 \times 10^{-5} \mathrm{~mol} / \mathrm{L}$ probe NaLy-CO for another $30 \mathrm{~min} . \lambda_{\mathrm{ex}}=405 \mathrm{~nm}, \lambda_{\mathrm{em}}=500 \sim 550 \mathrm{~nm}$, scale bar $=50 \mu \mathrm{m}$

在发现探针 NaLy-CO 能够追踪活细胞内外加的 $\mathrm{CO}$ 后, 继续探究探针 NaLy-CO 能否检测活细胞内源性 $\mathrm{CO}$. 研究表明生命体内的内源性 $\mathrm{CO}$ 来源于血红素氧合 酶(HO)催化分解血红素(Heme $)^{[2]}$, 因此利用外加的血红 素刺激细胞产生内源性 CO. 用 $1 \times 10^{-4} \mathrm{~mol} / \mathrm{L}$ 血红素 (图 8B)餒育细胞 $120 \mathrm{~min}$ 后进行苂光成像并没有苂光信
号产生. 然而, 先用 $1 \times 10^{-4} \mathrm{~mol} / \mathrm{L}$ 血红素刺激细胞 120 min 后继续使用探针 NaLy-CO 孵育 $30 \mathrm{~min}$ 后进行荧光 成像, 则显示出了较强的苂光信号(图 $8 \mathrm{E}$ ). 并且血红素 的刺激时间的越长，所观察到的绿色苂光发射信号越明 显(图 8E 和图 8H). 这些结果清楚地表明, 探针 NaLyCO 在活细胞内源性 CO 的检测领域拥有较大的应用潜 力.

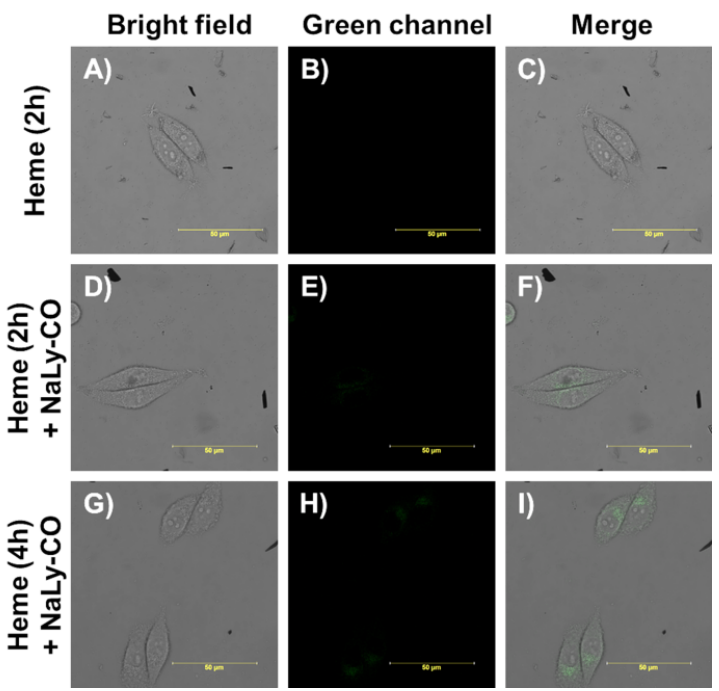

图 8 探针 NaLy-CO 在 HeLa 细胞中检测内源性 CO 的苂光 图像

Figure 8 Fluorescent images of probe NaLy-CO in HeLa cells for detecting endogenous $\mathrm{CO}$

$\mathrm{A} \sim \mathrm{C}$ : the HeLa cells were incubated with $1 \times 10^{-4} \mathrm{~mol} / \mathrm{L}$ Heme for $2 \mathrm{~h}$; $\mathrm{D} \sim \mathrm{F}$ : the HeLa cells were pre-cultured with $1 \times 10^{-4} \mathrm{~mol} / \mathrm{L}$ Heme for 2 $\mathrm{h}$, then cultured with $1 \times 10^{-5} \mathrm{~mol} / \mathrm{L}$ probe $\mathbf{N a L y}-\mathbf{C O}$ for another $30 \mathrm{~min}$; $\mathrm{G} \sim \mathrm{I}$ : the HeLa cells were pre-cultured with $1 \times 10^{-4} \mathrm{~mol} / \mathrm{L}$ Heme for 4 h, then cultured with $1 \times 10^{-5} \mathrm{~mol} / \mathrm{L}$ probe $\mathbf{N a L y}-\mathbf{C O}$ for another $30 \mathrm{~min}$. $\lambda_{\mathrm{ex}}=405 \mathrm{~nm}, \lambda_{\mathrm{em}}=500 \sim 550 \mathrm{~nm}$. scale bar $=50 \mu \mathrm{m}$

在成功验证了探针 NaLy-CO 能够进行细胞内 $\mathrm{CO}$ 的检测后，进行共定位实验以确定探针 NaLy-CO 在细 胞中的分布情况. 共定位实验数据显示(图 9 和表 1), 商 业溶酶体定位染料 Lyso Tracker Deep Red 的近红外苂光 与探针 NaLy-CO 的绿色菼光有很好的重叠, 计算出 Pearson 相关系数为 0.85. 相比之下, 探针 NaLy-CO 的 绿色苂光和商业线粒体定位染料 Mito Tracker Deep Red 的 Pearson 相关系数仅为 0.48 . 共定位成像实验结果表 明，探针 NaLy-CO 主要在细胞溶酶体上富集，能够检 测细胞溶酶体内的 CO.

表 1 探针 NALy-CO 与商业定位染料的共定位系数 Table 1 Co-localization coefficient of probe NALy-CO and commercial localized dye

\begin{tabular}{lcc}
\hline & $\begin{array}{l}\text { Lyso Tracker } \\
\text { Deep Red }\end{array}$ & Mito Tracker Red \\
\hline Pearson's correlation & 0.85 & 0.48 \\
\hline
\end{tabular}




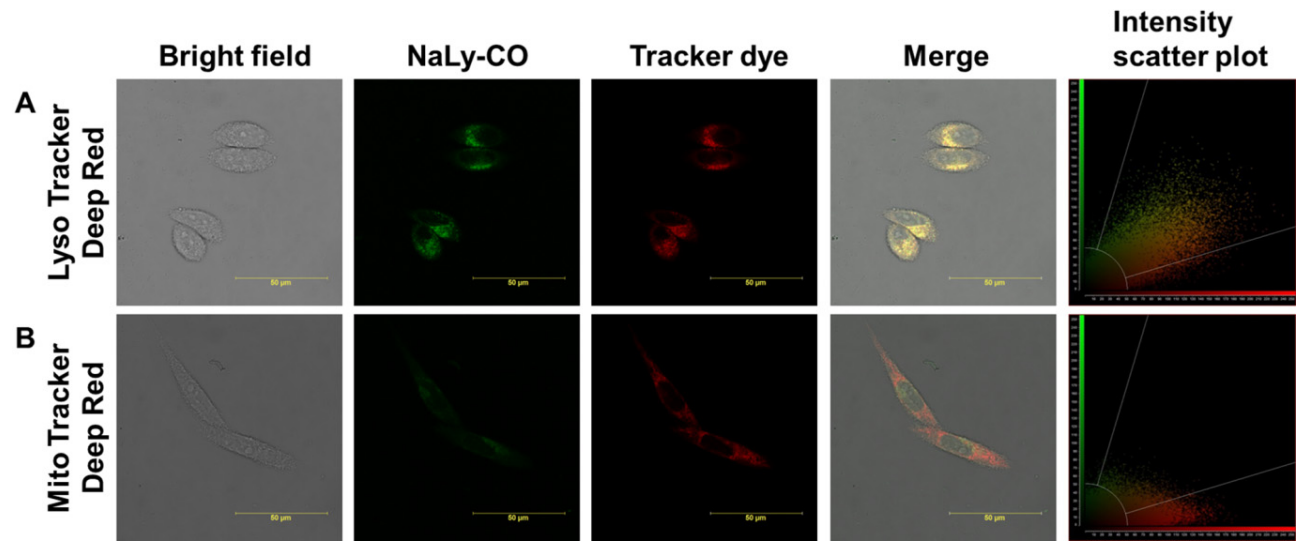

图 9 定位染料与外源性 CO 测试体系(探针 NaLy-CO 和 CORM-2)的共定位成像实验

Figure 9 Co-location imaging experiment of localized dyes and exogenous CO test system (probe NALy-CO and CORM-2) Co-location imaging experiments between Lyso Tracker Deep Red and probe NALy-CO; (B) Co-location imaging experiments between Mito Tracker Deep Red and probe NALy-CO. The green channel: $\lambda_{\mathrm{ex}}=405 \mathrm{~nm}, \lambda_{\mathrm{em}}=500 \sim 550 \mathrm{~nm}$; The deep red channel: $\lambda_{\mathrm{ex}}=647 \mathrm{~nm}, \lambda_{\mathrm{em}}=670 \sim 720 \mathrm{~nm}$. Scale bar: $50 \mu \mathrm{m}$

\section{2 结论}

通过合理的分析和设计，构建了第一个能够检测细 胞溶酶体内源性 CO 的苂光增强型探针 NaLy-CO. 起初 由于探针 NaLy-CO 上的硝基基团的强拉电子能力抑制 了 ICT 效应的产生, 使得探针几乎没有荧光信号发射. 当探针 NaLy-CO 与 $\mathrm{CO}$ 相互作用后，探针的硝基基团被 CO 还原为氨基基团, 因为 ICT 效应恢复而产生强烈的 荧光信号. 探针 NaLy-CO 在 PBS 溶液中对 CO 具有较 高的灵敏度和选择性. 此外, 探针 NaLy-CO 还具有检 测活细胞中外源性和内源性 CO 的能力. 重要的是, 共 定位成像实验表明该荧光探针主要在细胞溶酶体内富 集. 我们认为探针 NaLy-CO 可以作为细胞溶酶体 CO 的分子跟踪试剂, 具有潜在的应用价值.

\section{3 实验部分}

\section{1 仪器与试剂}

Bruker 的核磁共振波谱仪 (600 MHz); Waters Xevo G2-XS-QTOF 液质联用仪; Hitachi F-4700 荧光分光光度 计(日本 Hitachi); UV-2700 紫外可见分光光度计(日本岛 津公司); 柱层析硅胶(200 300 目)是青岛海洋化工厂 产品. 实验所用的试剂和溶剂均是国产分析纯或色谱 纯, 均不需要进一步处理.

\section{2 实验方法}

\subsection{1 探针 NaLy-CO 的合成}

根据参考文献[22]的方法进行化合物 $\mathbf{1}$ 的合成, 所 得产物无需进一步提纯直接使用. 将化合物 $1(0.24 \mathrm{~g}$, $1 \times 10^{-3} \mathrm{~mol}$ )加入到 $25 \mathrm{~mL}$ 的圆底烧瓶中, 再加入 2-(2氨基乙基)吡啶 $\left(0.183 \mathrm{~g}, 1.5 \times 10^{-3} \mathrm{~mol}\right)$ 和无水乙醇 $(3$ $\mathrm{mL}$ ), 在 $85{ }^{\circ} \mathrm{C}$ 下回流 $4 \mathrm{~h}$, 将反应液冷却至室温, 过滤,
滤饼用少量冰乙醇洗 3 次, 滤饼使用冰醋酸进行重结晶, 得到 $0.18 \mathrm{~g}$ 棕褐色固体, 产率 $52.1 \%$. m.p. 207.7 $208.2{ }^{\circ} \mathrm{C} ;{ }^{1} \mathrm{H}$ NMR $\left(600 \mathrm{MHz}, \mathrm{DMSO}-d_{6}\right) \delta: 9.42(\mathrm{~d}, J=$ $2.4 \mathrm{~Hz}, 1 \mathrm{H}), 8.85(\mathrm{~d}, J=2.4 \mathrm{~Hz}, 1 \mathrm{H}), 8.73(\mathrm{~d}, J=8.4 \mathrm{~Hz}$, $1 \mathrm{H}), 8.61(\mathrm{~d}, J=7.2 \mathrm{~Hz}, 1 \mathrm{H}), 8.44 \sim 8.46(\mathrm{~m}, 1 \mathrm{H}), 8.01(\mathrm{t}$, $J=7.8 \mathrm{~Hz}, 1 \mathrm{H}), 7.71(\mathrm{td}, J=7.8,1.8 \mathrm{~Hz}, 1 \mathrm{H}), 7.33(\mathrm{~d}, J=$ $7.8 \mathrm{~Hz}, 1 \mathrm{H}), 7.21 \sim 7.24(\mathrm{~m}, 1 \mathrm{H}), 4.38(\mathrm{t}, J=7.8 \mathrm{~Hz}, 2 \mathrm{H})$, $3.10(\mathrm{t}, J=7.8 \mathrm{~Hz}, 2 \mathrm{H}) ;{ }^{13} \mathrm{C}$ NMR $\left(150 \mathrm{MHz}\right.$, DMSO- $\left.d_{6}\right)$ $\delta: 163.08,162.58,158.93,149.52,146.24,137.08,136.76$, $134.34,131.29,130.15,129.89,129.70,124.38,123.68$, 123.23, 122.96, 122.15, 40.52, 35.76; HRMS calcd for $\mathrm{C}_{19} \mathrm{H}_{13} \mathrm{~N}_{3} \mathrm{O}_{4} 396.1686$, found 348.0979 .

\section{2 .2 光谱测试}

取适量的探针 NaLy-CO, 用二甲基亚砜溶解, 配成 $1 \times 10^{-3} \mathrm{~mol} / \mathrm{L}$ 的探针母液备用, 探针的测试浓度为 $1 \times 10^{-6} \mathrm{~mol} / \mathrm{L}$, 在测试选择性的过程中, GSH 的浓度为 $5 \times 10^{-3} \mathrm{~mol} / \mathrm{L}, L$-Cys 浓度为 $1 \times 10^{-3} \mathrm{~mol} / \mathrm{L}$, 其余的一 些氨基酸、阴阳离子以及活性氧等分析物质的测试浓度 均为 $5 \times 10^{-4} \mathrm{~mol} / \mathrm{L}, \mathrm{CORM}-2$ 的测试浓度为 $2 \times 10^{-4}$ $\mathrm{mol} / \mathrm{L}$. 测试过程中, 我们选用 PBS 缓冲溶液 $\left(1 \times 10^{-2}\right.$ $\mathrm{mol} / \mathrm{L}, \mathrm{pH} 7.4$, 体积分数 $10 \% \mathrm{DMSO}$ )作为测试溶液. 苂 光光谱的测试温度为 $37{ }^{\circ} \mathrm{C}$, 样品池的规格为 $1 \mathrm{~cm} \times 1$ $\mathrm{cm} \times 4 \mathrm{~cm}$, 选择激发波长为 $430 \mathrm{~nm}$, 选择狭缝为 $10 \mathrm{~nm}$.

\section{2 .3 细胞实验}

细胞毒性实验: 将培养好的 Hela 细胞接种于 96 孔 板当中, 每个孔的细胞溶液 $0.1 \mathrm{~mL}$, 将细胞培养过夜贴 壁后，向其中加入不同浓度的探针 NaLy-CO 培养基溶 液 $\left(0 \sim 5 \times 10^{-5} \mathrm{~mol} / \mathrm{L}\right)$, 在细胞培养箱内培养 $24 \mathrm{~h}$ 后, 向 每个孔内加入浓度为 $5 \times 10^{-2} \mathrm{~g} / \mathrm{L}$ 的噻唑蓝(MTT)培养 
基溶液，继续在细胞培养箱内孵育 $4 \mathrm{~h}$ 后，移除 96 孔板 内的培养基并向其中加入 $0.1 \mathrm{~mL}$ 的二甲基亚砜溶解生 成的紫色晶体, 在 $490 \mathrm{~nm}$ 下用酶标仪进行吸光度值测 试, 根据 Eq. 1 计算的细胞存活率.

$$
\text { Cell viability }(\%)=\left(\mathrm{OD}_{\text {sample }}-\mathrm{OD}_{\text {blank }}\right) /
$$$$
\left(\mathrm{OD}_{\text {control }}-\mathrm{OD}_{\text {blank }}\right) \times 100 \%
$$

$\mathrm{OD}_{\text {sample }}$ 指不同浓度探针孵育的细胞的吸光度, $\mathrm{OD}_{\text {control }}$ 指未加探针孵育的细胞的吸光度, $\mathrm{OD}_{\text {blank }}$ 为只包含培养 基的吸光度.

分三组实验进行细胞的外源性 CO 成像研究: 第一 组使用 $1 \times 10^{-5} \mathrm{~mol} / \mathrm{L}$ 探针 NaLy-CO 孵育细胞 $30 \mathrm{~min}$; 第二组使用 $1 \times 10^{-4} \mathrm{~mol} / \mathrm{L}$ CORM-2 孵育细胞 $30 \mathrm{~min}$; 第三组是先使用 $1 \times 10^{-4} \mathrm{~mol} / \mathrm{L}$ CORM-2 孵育细胞 30 $\min$ 后加入 $1 \times 10^{-5} \mathrm{~mol} / \mathrm{L}$ 探针 NaLy-CO 继续孵育细胞 $30 \mathrm{~min}$. 随后开始进行外源性 CO 成像实验. 成像条件: 激发波长为 $405 \mathrm{~nm}$ ，检测范围为 $500 \sim 550 \mathrm{~nm}$.

分三组实验进行细胞的内源性 CO 成像研究: 第一 组使用 $1 \times 10^{-4} \mathrm{~mol} / \mathrm{L}$ 血红素孵育细胞 $120 \mathrm{~min}$; 第二组 是先使用 $1 \times 10^{-4} \mathrm{~mol} / \mathrm{L}$ 血红素孵育细胞 $120 \mathrm{~min}$ 后加 入 $1 \times 10^{-5} \mathrm{~mol} / \mathrm{L}$ 探针 NaLy-CO 继续孵育细胞 $30 \mathrm{~min}$; 第三组是先使用 $1 \times 10^{-4} \mathrm{~mol} / \mathrm{L}$ 血红素孵育细胞 $240 \mathrm{~min}$ 后加入 $1 \times 10^{-5} \mathrm{~mol} / \mathrm{L}$ 探针 NaLy-CO 继续孵育细胞 30 min. 随后开始进行外源性 CO 成像实验. 成像条件: 激 发波长为 $405 \mathrm{~nm}$ ，检测范围为 $500 \sim 550 \mathrm{~nm}$.

细胞的共聚焦显微镜共定位实验：对于溶酶体共定 位实验, 先用 $5 \times 10^{-8} \mathrm{~mol} / \mathrm{L}$ 的溶酶体深红色菼光染料 (Lyso Tracker Deep Red)和 $1 \times 10^{-4} \mathrm{~mol} / \mathrm{L}$ CORM-2 孵育 $30 \mathrm{~min}$ 后, 加入 $1 \times 10^{-5} \mathrm{~mol} / \mathrm{L}$ 探针 NaLy-CO 继续孵育 细胞 $30 \mathrm{~min}$; 对于线粒体共定位实验, 先用 $1 \times 10^{-7}$ $\mathrm{mol} / \mathrm{L}$ 的线粒体深红色荧光染料(Mito Tracker Deep Red) 和 $1 \times 10^{-4} \mathrm{~mol} / \mathrm{L}$ CORM-2 孵育 $30 \mathrm{~min}$ 后, 加入 $1 \times$ $10^{-5} \mathrm{~mol} / \mathrm{L}$ 探针 NaLy-CO 继续孵育细胞 $30 \mathrm{~min}$. 随后开 始进行共定位成像实验. 成像条件: 对于探针 NaLy-CO, 激发波长为 $405 \mathrm{~nm}$, 检测范围为 500 550 $\mathrm{nm}$; 对于所商购的溶酶体深红色菼光染料和线粒体深 红色苂光染料，激发波长为 $640 \mathrm{~nm}$, 检测范围为 670 $720 \mathrm{~nm}$.

辅助材料(Supporting Information) 探针 NaLy-CO 的 ${ }^{1} \mathrm{H}$ NMR, ${ }^{13} \mathrm{C}$ NMR 以及 HRMS 谱图, 细胞毒性以及探
针与 CORM-2 反应后的高分辨质谱图谱. 这些材料可以 免费从本刊网站(http://sioc-journal.cn/)上下载.

\section{References}

[1] Prockop, L. D.; Chichkova, R. I. J. Neurol. Sci. 2007, 262, 122.

[2] Sjostrand T. J. Clin. Invest. 1949, 28, 201.

[3] Brune, B.; Ullrich, V. Mol. Pharmacol. 1987, 32, 497.

[4] Prabhakar, N. R.; Dinerman, J. L.; Agani, F. H.; Snyder, S. H. Proc. Natl. Acad. Sci. U. S. A. 1995, 92, 1994.

[5] Paro, F. M.; Steiner, A. A.; Branco, L. G. S. J. Therm. Biol. 2001 26,339 .

[6] Sato, K.; Balla, J.; Otterbein, L.; Smith, R. N.; Brouard, S.; Lin, Y.; Csizmadia, E.; Sevigny, J.; Robson, S. C.; Vercellotti, G.; Choi, A. M.; Bach, F. H.; Soares, M. P. J. Immunol. 2001, 166, 4185.

[7] Foresti, R.; Motterlini, R. Free Radical Res. 1999, 31, 459.

[8] Heinemann, S. H.; Hoshi, T.; Westerhausen, M.; Schiller, A. Chem. Commun. 2014, 50, 3644.

[9] Lee, I. T.; Luo, S.-F.; Lee, C.-W.; Wang, S.-W.; Lin, C.-C.; Chang, C.-C.; Chen, Y.-L.; Chau, L.-Y.; Yang, C.-M. Am. J. Pathol. 2009, $175,519$.

[10] Ryter, S. W.; Choi, A. M. K. Transl. Res. 2016, 167, 7.

[11] Wu, L.; Wang, R. Pharmacol. Rev. 2005, 57, 585.

[12] Schroeder, R. A.; Ewing, C. A.; Sitzmann, J. V.; Kuo, P. C. Dig. Dis. Sci. 2000, 45, 2405.

[13] Watts, C. Biochim. Biophys. Acta, Proteins Proteomics 2012, 1824, 14.

[14] Zhao, H. Traffic 2012, 13, 1307.

[15] Li, M.; Wang, Y.; Liu, G.; Lü, H.; Xing, G. Chin. J. Org. Chem., 2017, 37, 356 (in Chinese).

(李美含，王宇童，刘广建，吕海娟，邢国文，有机化学，2017, 37, 356.)

[16] Dell'Angelica, E. C. FASEB J. 2000, 14, 1265.

[17] Benito-Garagorri, D.; Puchberger, M.; Mereiter, K.; Kirchner, K. Angew. Chem., Int. Ed. 2008, 47, 9142.

[18] Kitagishi, H.; Minegishi, S.; Yumura, A.; Negi, S.; Taketani, S.; Amagase, Y.; Mizukawa, Y.; Urushidani, T.; Sugiura, Y.; Kano, K. J. Am. Chem. Soc. 2016, 138, 5417 .

[19] Park, S. S.; Kim, J.; Lee, Y. Anal. Chem. 2012, 84, 1792.

[20] Lv, B.; Xu, X.; Huang, D.; Wu, P.; Sheng, Z.; Liu, W.; Li, D.; Alharbi, N. S.; Zhang, K.; Wang, S. Chin. J. Org. Chem. 2018, 38, 3165 (in Chinese).

(吕卉, 徐学涛, 黄丹颖, 吴盼盼, 盛钊君, 刘文锋, 李冬利, Alharbi, N. S., 张焜, 王少华, 有机化学, 2018, 38, 3165.)

[21] Li, Y.; Lü, Z.; Liu, M.; Xing, G. Chin. J. Org. Chem. 2016, 36, 962 (in Chinese). (李杨洁，吕子奇，刘敏，邢国文，有机化学, 2016, 36, 962.)

[22] Luo, X.; Chen, M.; Yang, Q. Acta Chim. Sinica 2020, 78, 373 (in Chinese).

(罗兴荵, 陈敏文, 杨晴来, 化学学报, 2020, 78, 373.)

[23] Wei, C.; Zhang, P.; Li, X. Chin. J. Org. Chem. 2019, 39, 3375 (in Chinese).

(魏超, 张平竹, 李小六, 有机化学, 2019, 39, 3375.)

[24] Tafesh, A. M.; Weiguny, J. Chem. Rev. 1996, 96, 2035.

[25] Wang, K.-R.; Qian, F.; Wang, X.-M.; Tan, G.-H.; Rong, R.-X.; Cao, Z.-R.; Chen, H.; Zhang, P.-Z.; Li, X.-L. Chin. Chem. Lett. 2014, 25, 1087.

[26] Ma, C.; Wei, C.; Li, X.; Zheng, X.; Chen, B.; Wang, M.; Zhang, P.; Li, X. Dyes Pigm. 2019, 162, 624.

$(\mathrm{Lu}, \mathrm{Y}$. 